INPLASY

PROTOCOL

To cite: Zeng et al. Clinical Application of Tumor Vascular Disrupting Therapy: A Systematic Review. Inplasy protocol 202140111. doi: 10.37766/inplasy2021.4.0111

Received: 21 April 2021

Published: 22 April 2021

Corresponding author: Wen Zeng

zengw1010@163.com

Author Affiliation: National Center for International Research of Biotargeting Theranostics, Guangxi Medical University, Nanning, Guangxi, China.

Support: National Natural Science Found.

Review Stage at time of this submission: Data analysis.

Conflicts of interest:

None declared.

\section{Clinical Application of Tumor Vascular Disrupting Therapy: A Systematic Review}

Zeng, W1; Wu, P2; Zhong, LP3; He, J4; Huang, Y5.

Review question / Objective: Malignant tumor is a leading cause of death in every country of the world, which is seriously harmful to human health. In history, the approach to the development of anti-tumor therapy has mainly focused on the tumor cells themselves. Vascular disrupting therapy targeting blood vessels provides a new tumor treatment scheme, but its efficiency has not been scientifically and systematically evaluated. The purpose of this systematic review was to comprehensively evaluate the clinical efficacy of vascular disrupting therapy in tumor patients for the first time.

Condition being studied: Due to the abnormal structure and function of tumor vessels, tumor vascular disrupting therapy mainly destroys the existing blood vessels in tumor tissue and blocks the tumor vascular system quickly and selectively. Vascular disrupting agents (VDAs) can selectively target tumor blood vessels through a variety of ways, inhibit the blood flow in the tumor, lead to extensive secondary necrosis in the tumor, and keep the normal tissue relatively intact. Because VDAs is mainly targets the hypoxic area in the center of the tumor, the therapeutic effect of single-agent VDAs is often weak, leaving a viable tumor rim with an abundant supply of blood oxygen in this treatment. Therefore, VDA is often combined with other treatments for tumor rim cells. At present, there is no systematic review of vascular disrupting therapy in the treatment of tumors.

INPLASY registration number: This protocol was registered with the International Platform of Registered Systematic Review and Meta-Analysis Protocols (INPLASY) on 22 April 2021 and was last updated on 22 April 2021 (registration number INPLASY202140111).

\section{INTRODUCTION}

Review question / Objective: Malignant tumor is a leading cause of death in every country of the world, which is seriously harmful to human health. In history, the approach to the development of anti-tumor therapy has mainly focused on the tumor 
cells themselves. Vascular disrupting therapy targeting blood vessels provides a new tumor treatment scheme, but its efficiency has not been scientifically and systematically evaluated. The purpose of this systematic review was to comprehensively evaluate the clinical efficacy of vascular disrupting therapy in tumor patients for the first time.

Condition being studied: Due to the abnormal structure and function of tumor vessels, tumor vascular disrupting therapy mainly destroys the existing blood vessels in tumor tissue and blocks the tumor vascular system quickly and selectively. Vascular disrupting agents (VDAs) can selectively target tumor blood vessels through a variety of ways, inhibit the blood flow in the tumor, lead to extensive secondary necrosis in the tumor, and keep the normal tissue relatively intact. Because VDAs is mainly targets the hypoxic area in the center of the tumor, the therapeutic effect of single-agent VDAs is often weak, leaving a viable tumor rim with an abundant supply of blood oxygen in this treatment. Therefore, VDA is often combined with other treatments for tumor rim cells. At present, there is no systematic review of vascular discupting therapy in the treatment of tumors.

\section{METHODS}

Participant or population: We will include all participants with tumors. There are no restrictions on age, sex, course of disease and severity of the disease.

Intervention: This systematic review includes interventions using tumor vascular disrupting therapy or in combination with other therapies.

Comparator: Trials comparing VDAs treatment with standard treatment and / or placebo were included. A study of another active therapy combined with VDAs compared with the same active therapy alone was also included.

Study designs to be included: All clinical trials of vascular disrupting therapy in the treatment of tumors have no published status restrictions and no written language. Reviews, animal experiments, mechanism studies, laboratory studies and observational studies will be excluded.

Eligibility criteria: Selected according to the PICOS criteria (Participant, intervention, comparator, outcomes, and study design). For continuous data, the average difference or standardized average difference of $95 \%$ confidence interval will be used. For dichotomy result data, the risk ratio of $95 \%$ confidence interval will be used to evaluate the therapeutic effect.

Information sources: Before data extraction, a standard form will be prepared for data collection. The two researchers will independently extract the data included in the study and fill in the form. Any differences will be resolved by consensus. The following data will be extracted: first author, published year, drug, cancer type, number of patients, average age, gender ratio, treatment methods, outcomes or conclusions. If necessary, we will contact the original author for complete information.

Main outcome(s): Outcome index include recommended phase 2 trial dose, maximum tolerated dose, progression free survival, overall survival, overall response rate, time to progression, complete response, partial response, stable disease, progressive disease, tumor response rate, duration of response.

Quality assessment / Risk of bias analysis: We used the JADAD scale to evaluate the methodological quality of each clinical trial, and the higher the score, the better the reporting effect. Among them, the study of JADAD score $\geq 3$ is considered to be a high-quality final score $\geq 3$, which is considered to indicate a high-quality methodological report.

Strategy of data synthesis: Note Express was used for literature management.The data is extracted through a standardized Excel form. 
Subgroup analysis: Subgroup analysis based on clinical trial stages and VDAs types.

Sensitivity analysis: When sufficient research is available, a sensitivity analysis will be conducted to assess the robustness of the study, and missing data will be assessed based on sample size and methodological quality.

Country(ies) involved: China.

Keywords: clinical trials, vascular disrupting therapy, vascular disrupting agents.

Contributions of each author:

Author 1 - Wen Zeng.

Author 2 - Pan Wu.

Author 3 - Liping Zhong.

Author 4 - Jian He.

Author 5 - Yong Huang.

Conflicts of interest: Zeng wen has no conflicts to report. Huang yong has received research support from the Scientific and Technological Innovation Major Base of Guangxi, the State Project for Essential Drug Research and Development, Guangxi Key Research and Development Project. 